\title{
The Study of Fixed Point Theory for Various Multivalued Non-Self-Maps
}

\author{
Wei-Shih Du, ${ }^{1}$ Erdal Karapınar, ${ }^{2}$ and Naseer Shahzad ${ }^{3}$ \\ ${ }^{1}$ Department of Mathematics, National Kaohsiung Normal University, Kaohsiung 824, Taiwan \\ ${ }^{2}$ Department of Mathematics, Atilim University, Incek, 06836 Ankara, Turkey \\ ${ }^{3}$ Department of Mathematics, King Abdulaziz University, P.O. Box 80203, Jeddah 21859, Saudi Arabia
}

Correspondence should be addressed to Erdal Karapınar; erdalkarapinar@yahoo.com

Received 24 April 2013; Accepted 19 July 2013

Academic Editor: Abdellah Bnouhachem

Copyright (C) 2013 Wei-Shih Du et al. This is an open access article distributed under the Creative Commons Attribution License, which permits unrestricted use, distribution, and reproduction in any medium, provided the original work is properly cited.

\begin{abstract}
The basic motivation of this paper is to extend, generalize, and improve several fundamental results on the existence (and uniqueness) of coincidence points and fixed points for well-known maps in the literature such as Kannan type, Chatterjea type, Mizoguchi-Takahashi type, Berinde-Berinde type, Du type, and other types from the class of self-maps to the class of non-self-maps in the framework of the metric fixed point theory. We establish some fixed/coincidence point theorems for multivalued non-selfmaps in the context of complete metric spaces.
\end{abstract}

\section{Introduction}

During the last few decades, the celebrated Banach contraction principle, also known as the Banach fixed point theorem [1], has become one of the core topics of applied mathematical analysis. As a consequence, a number of generalizations, extensions, and improvement of the praiseworthy Banach contraction principle in various direction have been explored and reported by various authors; see, for example, [2-30] and the references therein. In parallel with the Banach contraction principle, Kannan [5] and Chatterjea [6] created, respectively, different type, fixed point theorems as follows.

Theorem 1 (Kannan). Let $(X, d)$ be a complete metric space, $T: X \rightarrow X$ is a single-valued map, and $\gamma \in[0,1 / 2)$. Assume that

$$
d(T x, T y) \leq \gamma[d(x, T x)+d(y, T y)] \quad \forall x, y \in X .
$$

Then $T$ has a unique fixed point in $X$.

Theorem 2 (Chatterjea). Let $(X, d)$ be a complete metric space, $T: X \rightarrow X$ is a single-valued map, and $\gamma \in[0,1 / 2)$. Assume that

$$
d(T x, T y) \leq \gamma[d(x, T y)+d(y, T x)] \quad \forall x, y \in X .
$$

Then $T$ has a unique fixed point in $X$.
The characterization of the renowned Banach fixed point theorem in the setting of multivalued maps is one of the most outstanding ideas of research in fixed point theory. The remarkable examples in this trend were given by Nadler [2], Mizoguchi and Takahashi [3], and M. Berinde and V. Berinde [4]. On the other hand, investigation of the existence of a fixed point of non-self-maps under certain condition is an interesting research subject of metric fixed point theory, see, for example, [19-27], and references therein.

The following attractive result was reported by $\mathrm{M}$. Berinde and V. Berinde [4] in 2007.

Theorem 3 (M. Berinde and V. Berinde). Let $(X, d)$ be a complete metric space, $T: X \rightarrow \mathscr{C} \mathscr{B}(X)$ a multivalued map, $\varphi:[0, \infty) \rightarrow[0,1)$ an $\mathscr{M} \mathscr{T}$-function (i.e., $\limsup _{s \rightarrow t^{+}} \varphi(s)<$ 1 for all $t \in[0, \infty)$ ), and $L \geq 0$. Assume that

$$
\begin{array}{r}
\mathscr{H}(T x, T y) \leq \varphi(d(x, y)) d(x, y)+L d(y, T x) \\
\forall x, y \in X .
\end{array}
$$

Then $T$ has a fixed point in $X$.

If we take $L=0$ in Theorem 3, then we conclude the remarkable result of Mizoguchi and Takahashi [3] which is a partial answer of problem 9 in [8]. 
Theorem 4 (Mizoguchi and Takahashi). Let $(X, d)$ be a complete metric space, $T: X \rightarrow \mathscr{C} \mathscr{B}(X)$ a multivalued map, and $\varphi:[0, \infty) \rightarrow[0,1)$ an $\mathscr{M} \mathcal{T}$-function. Assume that

$$
\mathscr{H}(T x, T y) \leq \varphi(d(x, y)) d(x, y) \quad \forall x, y \in X
$$

Then $T$ has a fixed point in $X$.

Recently, Du [12] established the following theorem which is an extension of Theorem 3 and hence Theorem 4.

Theorem $5(\mathrm{Du})$. Let $(X, d)$ be a complete metric space, $T$ : $X \rightarrow \mathscr{C} \mathscr{B}(X)$ a multivalued map, $\varphi:[0, \infty) \rightarrow[0,1)$ an $M \mathscr{T}$-function and $h: X \rightarrow[0, \infty)$ a function. Assume that

$$
\begin{array}{r}
\mathscr{H}(T x, T y) \leq \varphi(d(x, y)) d(x, y)+h(y) d(y, T x) \\
\forall x, y \in X .
\end{array}
$$

Then $T$ has a fixed point in $X$.

The basic objective of this paper is to investigate the existence of coincidence and fixed points of multivalued nonself-maps under the certain conditions in the setting of metric spaces. The presented results generalize, improve, and extend several crucial and notable results that examine the existence of the coincidence/fixed point of well-known maps such as Kannan type, Chatterjea type, Mizoguchi-Takahashi type, Berinde-Berinde type, Du type, and other types in the context of complete metric spaces.

\section{Preliminaries}

Let $(X, d)$ be a metric space. For each $x \in X$ and $A \subseteq$ $X$, let $d(x, A)=\inf _{y \in A} d(x, y)$. Denote by $\mathcal{N}(X)$ the class of all nonempty subsets of $X$ and $\mathscr{C} \mathscr{B}(X)$ the family of all nonempty closed and bounded subsets of $X$. A function $\mathscr{H}$ : $\mathscr{C} \mathscr{B}(X) \times \mathscr{C} \mathscr{B}(X) \rightarrow[0, \infty)$ defined by

$$
\mathscr{H}(A, B)=\max \left\{\sup _{x \in B} d(x, A), \sup _{x \in A} d(x, B)\right\}
$$

is said to be the Hausdorff metric on $\mathscr{C} \mathscr{B}(X)$ induced by the metric $d$ on $X$. It is also known that $(\mathscr{C} \mathscr{B}(X), \mathscr{H})$ is a complete metric space whenever $(X, d)$ is a complete metric space.

Let $K$ be a nonempty subset of $X, g: K \rightarrow X$ a singlevalued map, and $T: K \rightarrow \mathcal{N}(X)$ a multivalued map. A point $x$ in $X$ is a coincidence point of $g$ and $T$ if $g x \in T x$. If $g=i d$ is the identity map, then $x=g x \in T x$ and call $x$ a fixed point of $T$. The set of fixed points of $T$ and the set of coincidence point of $g$ and $T$ are denoted by $\mathscr{F}_{K}(T)$ and $\mathscr{C} O \mathscr{P}_{K}(g, T)$, respectively. In particular, if $K \equiv X$, we use $\mathscr{F}(T)$ and $\mathscr{C} \mathscr{O P}(g, T)$ instead of $\mathscr{F}_{K}(T)$ and $\mathscr{C} \mathscr{O} \mathscr{P}_{K}(g, T)$, respectively. Throughout this paper, we denote by $\mathbb{N}$, and $\mathbb{R}$, the set of positive integers and real numbers, respectively.

Let $f$ be a real-valued function defined on $\mathbb{R}$. For $c \in \mathbb{R}$, we recall that

$$
\limsup _{x \rightarrow c^{+}} f(x)=\inf _{\varepsilon>0} \sup _{c<x<c+\varepsilon} f(x) .
$$

Definition 6 (see [9-18]). A function $\varphi:[0, \infty) \rightarrow$ $[0,1)$ is said to be an $\mathscr{M T}$-function (or $\mathscr{R}$-function) if $\limsup _{s \rightarrow t^{+}} \varphi(s)<1$ for all $t \in[0, \infty)$.

It is evident that if $\varphi:[0, \infty) \rightarrow[0,1)$ is a nondecreasing function or a nonincreasing function, then $\varphi$ is an $\mathscr{M} \mathscr{T}$ function. So the set of $\mathscr{M} \mathscr{T}$-functions is a rich class. An example which is not an $\mathscr{M} \mathscr{T}$-function is given as follows. Let $\varphi:[0, \infty) \rightarrow[0,1)$ be defined by

$$
\varphi(t):= \begin{cases}\frac{\sin t}{t}, & \text { if } t \in\left(0, \frac{\pi}{2}\right] \\ 0, & \text { otherwise. }\end{cases}
$$

We note that $\varphi$ is not an $\mathscr{M} \mathscr{T}$-function, since $\lim \sup _{s \rightarrow 0^{+}} \varphi(s)=1$.

In what follows that, we recall some characterizations of $\mathscr{M} \mathscr{T}$-functions proved first by $\mathrm{Du}[12]$.

Theorem 7 (see [12]). Let $\varphi:[0, \infty) \rightarrow[0,1)$ be a function. Then the following statements are equivalent.

(a) $\varphi$ is an $\mathscr{M} \mathscr{T}$-function.

(b) For each $t \in[0, \infty)$, there exist $r_{t}^{(1)} \in[0,1)$ and $\varepsilon_{t}^{(1)}>0$ such that $\varphi(s) \leq r_{t}^{(1)}$ for all $s \in\left(t, t+\varepsilon_{t}^{(1)}\right)$.

(c) For each $t \in[0, \infty)$, there exist $r_{t}^{(2)} \in[0,1)$ and $\varepsilon_{t}^{(2)}>0$ such that $\varphi(s) \leq r_{t}^{(2)}$ for all $s \in\left[t, t+\varepsilon_{t}^{(2)}\right]$.

(d) For each $t \in[0, \infty)$, there exist $r_{t}^{(3)} \in[0,1)$ and $\varepsilon_{t}^{(3)}>0$ such that $\varphi(s) \leq r_{t}^{(3)}$ for all $s \in\left(t, t+\varepsilon_{t}^{(3)}\right]$.

(e) For each $t \in[0, \infty)$, there exist $r_{t}^{(4)} \in[0,1)$ and $\varepsilon_{t}^{(4)}>0$ such that $\varphi(s) \leq r_{t}^{(4)}$ for all $s \in\left[t, t+\varepsilon_{t}^{(4)}\right)$.

(f) For any nonincreasing sequence $\left\{x_{n}\right\}_{n \in \mathbb{N}}$ in $[0, \infty)$, one has $0 \leq \sup _{n \in \mathbb{N}} \varphi\left(x_{n}\right)<1$.

(g) $\varphi$ is a function of contractive factor; that is, for any strictly decreasing sequence $\left\{x_{n}\right\}_{n \in \mathbb{N}}$ in $[0, \infty)$, we have $0 \leq \sup _{n \in \mathbb{N}} \varphi\left(x_{n}\right)<1$.

\section{Existence Theorems of Coincidence Points and Fixed Points for Multivalued Non-Self- Maps of Kannan Type and Chatterjea Type}

In this section, we prove the existence of coincidence points and fixed points of multivalued non-self-maps of Kannan type and Chatterjea type. For this purpose, we first established a new intersection theorem of $\mathscr{C} \mathscr{O} \mathscr{P}_{K}(g, T)$ and $\mathscr{F}_{K}(T)$ for multivalued non-self-maps in complete metric spaces.

Theorem 8. Let $(X, d)$ be a complete metric space, $K a$ nonempty closed subset of $X, T: K \rightarrow \mathscr{C} \mathscr{B}(X)$ a multivalued map and $g: K \rightarrow X$ a continuous self-map. Suppose that

(D1) $T x \cap K \neq \emptyset$ for all $x \in K$,

(D2) $T x \cap K$ is g-invariant (i.e., $g(T x \cap K) \subseteq T x \cap K)$ for each $x \in K$, 
(D3) there exist a function $h: K \rightarrow[0, \infty)$ and $\gamma \in[0,1 / 2)$ such that

$$
\begin{gathered}
\mathscr{H}(T x, T y \cap K) \\
\leq \gamma[d(x, T x \cap K)+d(y, T x \cap K)+d(y, T y \cap K)] \\
+h(y) d(g y, T x \cap K) \\
\forall x, y \in K .
\end{gathered}
$$

$$
\text { Then } \mathscr{C} \mathscr{O} \mathscr{P}_{K}(g, T) \cap \mathscr{F}_{K}(T) \neq \emptyset \text {. }
$$

Proof. Since $K$ a nonempty closed subset of $X$ and $X$ is complete, $(K, d)$ is also a complete metric space. Let $x \in K$. Put $k=\gamma /(1-\gamma)$ and $\lambda=(1+k) / 2$. So $0 \leq k<\lambda<1$. Let $y \in T x \cap K$ be arbitrary. Then $d(y, T x \cap K))=0$. By (D2), we have $d(g y, T x \cap K)=0$. Hence (9) implies

$$
\begin{aligned}
& \mathscr{H}(T x, T y \cap K) \\
& \leq \gamma[d(x, T x \cap K)+\mathscr{H}(T x, T y \cap K)] \\
& \forall y \in T x \cap K .
\end{aligned}
$$

Inequality (10) shows that

$$
\begin{aligned}
& d(y, T y \cap K) \leq \mathscr{H}(T x, T y \cap K) \\
& \leq k d(x, T x \cap K)<\lambda d(x, y) \\
& \forall y \in T x \cap K .
\end{aligned}
$$

Let $x \in K$ be given. Take $x_{1}=x$. By (D1), $T x_{1} \cap K \neq \emptyset$. Choose $x_{2} \in T x_{1} \cap K$. If $x_{2}=x_{1}$, then $x_{1} \in \mathscr{F}_{K}(T)$ and hence $g x_{1} \in$ $T x_{1}$ from (D2). Hence $x_{1} \in \mathscr{C} \mathscr{O} \mathscr{P}_{K}(g, T) \cap \mathscr{F}_{K}(T)$ and the proof is finished. Otherwise, if $x_{2} \neq x_{1}$, then $d\left(x_{1}, x_{2}\right)>0$. By (11), we have

$$
d\left(x_{2}, T x_{2} \cap K\right)<\lambda d\left(x_{1}, x_{2}\right),
$$

which implies that there exists $x_{3} \in T x_{2} \cap K$ such that

$$
d\left(x_{2}, x_{3}\right)<\lambda d\left(x_{1}, x_{2}\right) .
$$

Next, by (11) again, there exists $x_{4} \in T x_{3} \cap K$ such that

$$
d\left(x_{3}, x_{4}\right)<\lambda d\left(x_{2}, x_{3}\right) .
$$

By induction, we can obtain a sequence $\left\{x_{n}\right\}$ in $K$ satisfying

$$
\begin{gathered}
x_{n+1} \in T x_{n} \cap K, \\
d\left(x_{n+1}, x_{n+2}\right)<\lambda d\left(x_{n}, x_{n+1}\right) .
\end{gathered}
$$

By (16), we have

$$
\begin{aligned}
d\left(x_{n+1}, x_{n+2}\right) & <\lambda d\left(x_{n}, x_{n+1}\right) \\
& <\lambda^{2} d\left(x_{n-1}, x_{n}\right) \\
& <\cdots \\
& <\lambda^{n} d\left(x_{1}, x_{2}\right), \quad \text { for } n \in \mathbb{N} .
\end{aligned}
$$

Let $\rho_{n}=\left(\lambda^{n-1} /(1-\lambda)\right) d\left(x_{1}, x_{2}\right), n \in \mathbb{N}$. For $m, n \in \mathbb{N}$ with $m>n$, we have

$$
d\left(x_{n}, x_{m}\right) \leq \sum_{j=n}^{m-1} d\left(x_{j}, x_{j+1}\right)<\rho_{n} .
$$

Since $0<\lambda<1, \lim _{n \rightarrow \infty} \rho_{n}=0$ and hence $\lim _{n \rightarrow \infty} \sup \left\{d\left(x_{n}, x_{m}\right): m>n\right\}=0$. This proves that $\left\{x_{n}\right\}$ is a Cauchy sequence in $K$. By the completeness of $K$, there exists $v \in K$ such that $x_{n} \rightarrow v$ as $n \rightarrow \infty$. By (15) and (D2), we have

$$
g x_{n+1} \in T x_{n} \cap K \quad \text { for each } n \in \mathbb{N} \text {. }
$$

Since $g$ is continuous and $\lim _{n \rightarrow \infty} x_{n}=v$, we have

$$
\lim _{n \rightarrow \infty} g x_{n}=g v
$$

Since the function $x \mapsto d(x, T v)$ is continuous, by (9), (15), (19), and (20), we get

$$
\begin{gathered}
d(v, T v \cap K) \\
=\lim _{n \rightarrow \infty} d\left(x_{n+1}, T v \cap K\right) \\
\leq \lim _{n \rightarrow \infty} \mathscr{H}\left(T x_{n}, T v \cap K\right) \\
\leq \lim _{n \rightarrow \infty}\left\{v \left[d\left(x_{n}, T x_{n} \cap K\right)+d\left(v, T x_{n} \cap K\right)\right.\right. \\
+d(v, T v \cap K)] \\
\left.+h(v) d\left(g v, T x_{n} \cap K\right)\right\} \\
\leq \lim _{n \rightarrow \infty}\left\{\gamma \left[d\left(x_{n}, x_{n+1}\right)\right.\right. \\
\left.+d\left(v, x_{n+1}\right)+d(v, T v \cap K)\right] \\
\left.+h(v) d\left(g v, g x_{n+1}\right)\right\}
\end{gathered}
$$

which implies $d(v, T v \cap K)=0$. By the closedness of $T v$, we have $v \in T v \cap K$. From (D2), $g v \in T v \cap K \subseteq T v$. Hence we verify $v \in \mathscr{C} \mathscr{O} \mathscr{P}_{K}(g, T) \cap \mathscr{F}_{K}(T)$. The proof is complete.

Theorem 9. In Theorem 8, if condition (D3) is replaced with one of the following conditions:

(K1) there exist a function $h: K \rightarrow[0, \infty)$ and $\gamma \in[0,1 / 2)$ such that

$$
\begin{aligned}
\mathscr{H}(T x, T y \cap K) & \\
\leq & \gamma[d(x, T x \cap K) \\
& +d(y, T x \cap K)+d(y, T y \cap K)] \\
& +h(y) d(g y, T x) \quad \forall x, y \in K,
\end{aligned}
$$


(K2) there exist a function $h: K \rightarrow[0, \infty)$ and $\gamma \in[0,1 / 2)$ such that

$$
\begin{aligned}
\mathscr{H}(T x, T y \cap K) & \\
\leq & \gamma[d(x, T x \cap K)+d(y, T x)+d(y, T y \cap K)] \\
& +h(y) d(g y, T x \cap K) \quad \forall x, y \in K,
\end{aligned}
$$

(K3) there exist a function $h: K \rightarrow[0, \infty)$ and $\gamma \in[0,1 / 2)$ such that

$\mathscr{H}(T x, T y \cap K)$

$$
\begin{aligned}
\leq & \gamma[d(x, T x \cap K)+d(y, T x)+d(y, T y \cap K)] \\
& +h(y) d(g y, T x) \quad \forall x, y \in K,
\end{aligned}
$$

(K4) there exist a function $h: K \rightarrow[0, \infty)$ and $\gamma \in[0,1 / 2)$ such that

$\mathscr{H}(T x, T y \cap K)$

$$
\begin{aligned}
\leq & \gamma[d(x, T x)+d(y, T x \cap K)+d(y, T y \cap K)] \\
& +h(y) d(g y, T x \cap K) \quad \forall x, y \in K,
\end{aligned}
$$

(K5) there exist a function $h: K \rightarrow[0, \infty)$ and $\gamma \in[0,1 / 2)$ such that

$\mathscr{H}(T x, T y \cap K)$

$$
\begin{aligned}
\leq & \gamma[d(x, T x)+d(y, T x \cap K)+d(y, T y \cap K)] \\
& +h(y) d(g y, T x) \quad \forall x, y \in K,
\end{aligned}
$$

(K6) there exist a function $h: K \rightarrow[0, \infty)$ and $\gamma \in[0,1 / 2)$ such that

$$
\begin{aligned}
\mathscr{H}(T x, T y \cap K) & \\
\leq & \gamma[d(x, T x)+d(y, T x)+d(y, T y \cap K)] \\
& +h(y) d(g y, T x \cap K) \quad \forall x, y \in K,
\end{aligned}
$$

(K7) there exist a function $h: K \rightarrow[0, \infty)$ and $\gamma \in[0,1 / 2)$ such that

$$
\begin{aligned}
\mathscr{H}(T x, T y \cap K) & \\
\leq & \gamma[d(x, T x)+d(y, T x)+d(y, T y \cap K)] \\
& +h(y) d(g y, T x) \quad \forall x, y \in K .
\end{aligned}
$$

Then $\mathscr{C} \mathscr{O} \mathscr{P}_{K}(g, T) \cap \mathscr{F}_{K}(T) \neq \emptyset$.

Proof. It is obvious that any of these conditions (K1)-(K7) implies condition (D3) as in Theorem 8. So the desired conclusion follows from Theorem 8 immediately.

The following fixed point theorem for multivalued nonself-maps of generalized Kannan type can be established immediately from Theorem 9 for $g \equiv i d$ (the identity mapping).
Theorem 10. Let $(X, d)$ be a complete metric space, $K$ a nonempty closed subset of $X$, and $T: K \rightarrow \mathscr{C} \mathscr{B}(X)$ a multivalued map. Suppose that $T x \cap K \neq \emptyset$ for all $x \in K$ and one of the following conditions holds:

(P1) there exist a function $h: K \rightarrow[0, \infty)$ and $\gamma \in[0,1 / 2)$ such that

$$
\begin{aligned}
\mathscr{H}(T x, T y \cap K) & \\
\leq & \gamma[d(x, T x \cap K)+d(y, T x \cap K)+d(y, T y \cap K)] \\
& +h(y) d(y, T x \cap K) \quad \forall x, y \in K
\end{aligned}
$$

(P2) there exist a function $h: K \rightarrow[0, \infty)$ and $\gamma \in[0,1 / 2)$ such that

$$
\begin{aligned}
\mathscr{H}(T x, T y \cap K) & \\
\leq & \gamma[d(x, T x \cap K)+d(y, T x \cap K)+d(y, T y \cap K)] \\
& +h(y) d(y, T x) \quad \forall x, y \in K,
\end{aligned}
$$

(P3) there exist a function $h: K \rightarrow[0, \infty)$ and $\gamma \in[0,1 / 2)$ such that

$$
\begin{aligned}
\mathscr{H}(T x, T y \cap K) & \\
\leq & \gamma[d(x, T x \cap K)+d(y, T x)+d(y, T y \cap K)] \\
& +h(y) d(y, T x \cap K) \quad \forall x, y \in K,
\end{aligned}
$$

(P4) there exist a function $h: K \rightarrow[0, \infty)$ and $\gamma \in[0,1 / 2)$ such that

$$
\begin{aligned}
\mathscr{H}(T x, T y \cap K) & \\
\leq & \gamma[d(x, T x \cap K)+d(y, T x)+d(y, T y \cap K)] \\
& +h(y) d(y, T x) \quad \forall x, y \in K,
\end{aligned}
$$

(P5) there exist a function $h: K \rightarrow[0, \infty)$ and $\gamma \in[0,1 / 2)$ such that

$$
\begin{aligned}
\mathscr{H}(T x, T y \cap K) & \\
\leq & \gamma[d(x, T x)+d(y, T x \cap K)+d(y, T y \cap K)] \\
& +h(y) d(y, T x \cap K) \quad \forall x, y \in K,
\end{aligned}
$$

(P6) there exist a function $h: K \rightarrow[0, \infty)$ and $\gamma \in[0,1 / 2)$ such that

$$
\begin{aligned}
\mathscr{H}(T x, T y \cap K) & \\
\leq & \gamma[d(x, T x)+d(y, T x \cap K)+d(y, T y \cap K)] \\
& +h(y) d(y, T x) \quad \forall x, y \in K,
\end{aligned}
$$


(P7) there exist a function $h: K \rightarrow[0, \infty)$ and $\gamma \in[0,1 / 2)$ such that

$$
\begin{aligned}
\mathscr{H}(T x, T y \cap K) & \\
\leq & \gamma[d(x, T x)+d(y, T x)+d(y, T y \cap K)] \\
& +h(y) d(y, T x \cap K) \quad \forall x, y \in K,
\end{aligned}
$$

(P8) there exist a function $h: K \rightarrow[0, \infty)$ and $\gamma \in[0,1 / 2)$ such that

$$
\begin{aligned}
& \mathscr{H}(T x, T y \cap K) \\
& \leq \gamma[d(x, T x)+d(y, T x)+d(y, T y \cap K)] \\
&+h(y) d(y, T x) \quad \forall x, y \in K .
\end{aligned}
$$

Then $\mathscr{F}_{K}(T) \neq \emptyset$.

As a consequence of Theorem 10, we obtain the following generalized Kannan type fixed point theorems for multivalued maps.

Corollary 11. Let $(X, d)$ be a complete metric space, $K a$ nonempty closed subset of $X$, and $T: K \rightarrow \mathscr{C} \mathscr{B}(X) a$ multivalued map. Suppose that $T x \cap K \neq \emptyset$ for all $x \in K$ and there exists $\gamma \in[0,1 / 2)$ such that

$$
\begin{array}{r}
\mathscr{H}(T x, T y \cap K) \leq \gamma[d(x, T x \cap K)+d(y, T y \cap K)] \\
\forall x, y \in K .
\end{array}
$$

Then $\mathscr{F}_{K}(T) \neq \emptyset$.

Remark 12. (a) If $K=X$ in Corollary 11, then we can obtain a multivalued version of Kannan's fixed point theorem [5].

(b) Theorems 8-10 and Corollary 11 all extend and generalize Kannan's fixed point theorem.

Theorem 13. Let $(X, d)$ be a complete metric space, $K$ a nonempty closed subset of $X, T: K \rightarrow \mathscr{C} \mathscr{B}(X)$ a multivalued map, and $g: K \rightarrow X$ a continuous self-map. Suppose that conditions (D1) and (D2) as in Theorem 8 hold. If there exist $h: K \rightarrow[0, \infty)$ and $\gamma \in[0,1 / 2)$ such that

$$
\begin{aligned}
& \mathscr{H}(T x, T y \cap K) \\
& \leq \alpha[d(x, T y \cap K)+d(y, T x \cap K)] \\
&+h(y) d(g y, T x \cap K) \quad \forall x, y \in K .
\end{aligned}
$$

Then $\mathscr{C} \mathscr{O} \mathscr{P}_{K}(g, T) \cap \mathscr{F}_{K}(T) \neq \emptyset$.

Proof. Let $x \in K$. Since $\alpha \in[0,1 / 2)$, by the denseness of $\mathbb{R}$, we can find $\beta>0$ such that $\alpha<\beta<1 / 2$. Let $y \in T x \cap K$ be arbitrary. Then $d(y, T x \cap K)=0$. By (D2), we have $d(g y, T x \cap$ $K)=0$. Hence (38) has been reduced to

$$
\begin{aligned}
d(y, T y \cap K) & \leq \mathscr{H}(T x, T y \cap K) \\
& \leq \alpha d(x, T y \cap K) \\
& <\beta d(x, T y \cap K) \quad \forall y \in T x \cap K .
\end{aligned}
$$

Let $x \in K$ be given. Take $x_{1}=x$. By (D1), Tx $x_{1} \cap K \neq \emptyset$. Choose $x_{2} \in T x_{1} \cap K$. If $x_{2}=x_{1}$, then $x_{1} \in \mathscr{F}_{K}(T)$ and hence $g x_{1} \in$ $T x_{1}$ from (D2). Hence $x_{1} \in \mathscr{C} \mathscr{O} \mathscr{P}_{K}(g, T) \cap \mathscr{F}_{K}(T)$ and the proof is finished. Otherwise, if $x_{2} \neq x_{1}$, then $d\left(x_{1}, x_{2}\right)>0$. By (39), we have

$$
d\left(x_{2}, T x_{2} \cap K\right)<\beta d\left(x_{1}, T x_{2} \cap K\right),
$$

which implies that there exists $x_{3} \in T x_{2} \cap K$ such that

$$
\begin{aligned}
d\left(x_{2}, x_{3}\right) & <\beta d\left(x_{1}, T x_{2} \cap K\right) \\
& \leq \beta d\left(x_{1}, x_{3}\right) \\
& \leq \beta\left[d\left(x_{1}, x_{2}\right)+d\left(x_{2}, x_{3}\right)\right] .
\end{aligned}
$$

Let $\gamma=\beta /(1-\beta)$. Then $\gamma \in(0,1)$ and the last inequality implies

$$
d\left(x_{2}, x_{3}\right)<\gamma d\left(x_{1}, x_{2}\right) .
$$

Continuing in this way, we can construct inductively a sequence $\left\{x_{n}\right\}_{n \in \mathbb{N}}$ in $K$ satisfying

$$
\begin{gathered}
x_{n+1} \in T x_{n} \cap K, \\
d\left(x_{n+1}, x_{n+2}\right)<\gamma d\left(x_{n}, x_{n+1}\right)
\end{gathered}
$$

for each $n \in \mathbb{N}$. Using a similar argument as in the proof of Theorem 8, we have the following:

(i) $x_{n+1} \in T x_{n} \cap K$;

(ii) $\left\{x_{n}\right\}$ is a Cauchy sequence in $K$;

(iii) there exists $v \in K$ such that $x_{n} \rightarrow v$ as $n \rightarrow \infty$;

(iv) $g x_{n+1} \in T x_{n} \cap K$ for each $n \in \mathbb{N}$;

(v) $\lim _{n \rightarrow \infty} g x_{n}=g v$.

By (38), we get

$$
\begin{aligned}
& d(v, T v \cap K) \\
& =\lim _{n \rightarrow \infty} d\left(x_{n+1}, T v \cap K\right) \\
& \leq \lim _{n \rightarrow \infty} \mathscr{H}\left(T x_{n}, T v \cap K\right) \\
& \leq \lim _{n \rightarrow \infty}\left\{\alpha\left[d\left(x_{n}, T v \cap K\right)+d\left(v, T x_{n} \cap K\right)\right]\right. \\
& \left.\quad+h(v) d\left(g v, T x_{n} \cap K\right)\right\} \\
& \leq \lim _{n \rightarrow \infty}\left\{\alpha\left[d\left(x_{n}, T v \cap K\right)+d\left(v, x_{n+1}\right)\right]\right. \\
& \left.\quad+h(v) d\left(g v, g x_{n+1}\right)\right\} \\
& =\alpha d(v, T v \cap K),
\end{aligned}
$$

which implies $d(v, T v \cap K)=0$. By the closedness of $T v$, we have $v \in T v \cap K$. By (D2), gv $\in T v \cap K \subseteq T v$ and hence $v \in \mathscr{C} \mathscr{O} \mathscr{P}_{K}(g, T) \cap \mathscr{F}_{K}(T)$. The proof is complete. 
Theorem 14. In Theorem 13, if inequality (38) is replaced with one of the following inequalities:

(C1)

$$
\begin{aligned}
\mathscr{H}(T x, T y \cap K) & \\
\leq & \alpha[d(x, T y \cap K)+d(y, T x \cap K)] \\
& +h(y) d(g y, T x) \quad \forall x, y \in K,
\end{aligned}
$$

(C2)

$$
\begin{aligned}
\mathscr{H}(T x, T y \cap K) & \\
\leq & \alpha[d(x, T y)+d(y, T x \cap K)] \\
& +h(y) d(g y, T x \cap K) \quad \forall x, y \in K,
\end{aligned}
$$

(C3)

$$
\begin{aligned}
\mathscr{H}(T x, T y \cap K) & \\
\leq & \alpha[d(x, T y)+d(y, T x \cap K)] \\
& +h(y) d(g y, T x) \quad \forall x, y \in K,
\end{aligned}
$$

(C4)

$$
\begin{aligned}
\mathscr{H}(T x, T y \cap K) & \\
\leq & \alpha[d(x, T y \cap K)+d(y, T x)] \\
& +h(y) d(g y, T x \cap K) \quad \forall x, y \in K,
\end{aligned}
$$

(C5)

$$
\begin{aligned}
\mathscr{H}(T x, T y \cap K) & \\
\leq & \alpha[d(x, T y \cap K)+d(y, T x)] \\
& +h(y) d(g y, T x) \quad \forall x, y \in K,
\end{aligned}
$$

(C6)

$$
\begin{aligned}
\mathscr{H}(T x, T y \cap K) & \\
\leq & \alpha[d(x, T y)+d(y, T x)] \\
& +h(y) d(g y, T x \cap K) \quad \forall x, y \in K,
\end{aligned}
$$

(C7)

$$
\begin{aligned}
\mathscr{H}(T x, T y \cap K) & \\
\leq & \alpha[d(x, T y)+d(y, T x)] \\
& +h(y) d(g y, T x) \quad \forall x, y \in K,
\end{aligned}
$$

then $\mathscr{C} \mathscr{O} \mathscr{P}_{K}(g, T) \cap \mathscr{F}_{K}(T) \neq \emptyset$.

Applying Theorem 14, we can prove the following fixed point theorems for multivalued maps of generalized Chatterjea type.

Theorem 15. Let $(X, d)$ a complete metric space, $K a$ nonempty closed subset of $X$, and $T: K \rightarrow \mathscr{C} \mathscr{B}(X)$ a multivalued map. Suppose that $T x \cap K \neq \emptyset$ for all $x \in K$ and one of the following conditions holds:

(Q1) there exist a function $h: K \rightarrow[0, \infty)$ and $\gamma \in[0,1 / 2)$ such that

$$
\begin{aligned}
\mathscr{H}(T x, T y \cap K) & \\
\leq & \alpha[d(x, T y \cap K)+d(y, T x \cap K)] \\
& +h(y) d(y, T x \cap K) \quad \forall x, y \in K,
\end{aligned}
$$

(Q2) there exist a function $h: K \rightarrow[0, \infty)$ and $\gamma \in[0,1 / 2)$ such that

$$
\begin{aligned}
\mathscr{H}(T x, T y \cap K) & \\
\leq & \alpha[d(x, T y \cap K)+d(y, T x \cap K)] \\
& +h(y) d(y, T x) \quad \forall x, y \in K,
\end{aligned}
$$

(Q3) there exist a function $h: K \rightarrow[0, \infty)$ and $\gamma \in[0,1 / 2)$ such that

$$
\begin{aligned}
\mathscr{H}(T x, T y \cap K) & \\
\leq & \alpha[d(x, T y)+d(y, T x \cap K)] \\
& +h(y) d(y, T x \cap K) \quad \forall x, y \in K,
\end{aligned}
$$

(Q4) there exist a function $h: K \rightarrow[0, \infty)$ and $\gamma \in[0,1 / 2)$ such that

$$
\begin{aligned}
\mathscr{H}(T x, T y \cap K) & \\
\leq & \alpha[d(x, T y)+d(y, T x \cap K)] \\
& +h(y) d(y, T x) \quad \forall x, y \in K,
\end{aligned}
$$

(Q5) there exist a function $h: K \rightarrow[0, \infty)$ and $\gamma \in[0,1 / 2)$ such that

$$
\begin{aligned}
\mathscr{H}(T x, T y \cap K) & \\
\leq & \alpha[d(x, T y \cap K)+d(y, T x)] \\
& +h(y) d(y, T x \cap K) \quad \forall x, y \in K,
\end{aligned}
$$

(Q6) there exist a function $h: K \rightarrow[0, \infty)$ and $\gamma \in[0,1 / 2)$ such that

$$
\begin{aligned}
\mathscr{H}(T x, T y \cap K) & \\
\leq & \alpha[d(x, T y \cap K)+d(y, T x)] \\
& +h(y) d(y, T x) \quad \forall x, y \in K,
\end{aligned}
$$

(Q7) there exist a function $h: K \rightarrow[0, \infty)$ and $\gamma \in[0,1 / 2)$ such that

$$
\begin{aligned}
\mathscr{H}(T x, T y \cap K) & \\
\leq & \alpha[d(x, T y)+d(y, T x)] \\
& +h(y) d(y, T x \cap K) \quad \forall x, y \in K,
\end{aligned}
$$


(Q8) there exist a function $h: K \rightarrow[0, \infty)$ and $\gamma \in[0,1 / 2)$ such that

$$
\begin{aligned}
\mathscr{H}(T x, T y \cap K) & \\
\leq & \alpha[d(x, T y)+d(y, T x)] \\
& +h(y) d(y, T x) \quad \forall x, y \in K .
\end{aligned}
$$

Then $\mathscr{F}_{K}(T) \neq \emptyset$.

The following result is a generalized Chatterjea's type fixed point theorem for multivalued maps in complete metric spaces.

Corollary 16. Let $(X, d)$ be a complete metric space, $K$ a nonempty closed subset of $X$, and $T: K \rightarrow \mathscr{B} \mathscr{C}(X)$ a multivalued map. Suppose that $T x \cap K \neq \emptyset$ for all $x \in K$ and there exists $\gamma \in[0,1 / 2)$ such that

$$
\mathscr{H}(T x, T y \cap K) \leq \alpha[d(x, T y)+d(y, T x)] \quad \forall x, y \in X .
$$

Then $\mathscr{F}_{K}(T) \neq \emptyset$.

Remark 17. (a) If $K=X$ in Corollary 16 , then we can obtain a multivalued version of Chatterjea's fixed point theorem [6].

(b) Theorems 13-15 and Corollary 16 all improve and generalize Chatterjea's fixed point theorem.

\section{New Coincidence and Fixed Point Results for Various Multivalued Non-Self-Maps: Mizoguchi-Takahashi Type, Berinde-Berinde Type, and Du Type}

In this section, we prove some coincidence and fixed point theorems for multivalued non-self-maps of MizoguchiTakahashi type, Berinde-Berinde type, and Du type.

Recall first the following auxiliary result.

Lemma 18 (see $[9$, Lemma 2.1]). Let $\varphi:[0, \infty) \rightarrow[0,1)$ be an $\mathscr{M T}$-function. Suppose that $\kappa:[0, \infty) \rightarrow[0,1)$ is defined by $\kappa(t)=(1+\varphi(t)) / 2$. Then, $\kappa$ is also an $\mathscr{M} \mathscr{T}$-function.

Theorem 19. Let $(X, d)$ be a complete metric space, $K$ a nonempty closed subset of $X, T: K \rightarrow \mathscr{B} \mathscr{C}(X)$ a multivalued map, and $g: K \rightarrow X$ be a continuous self-map. Suppose that conditions (D1) and (D2) as in Theorem 8 hold. If there exist an $\mathscr{M} \mathscr{T}$-function $\varphi:[0, \infty) \rightarrow[0,1)$ and a function $h: K \rightarrow[0, \infty)$ such that

$$
\begin{aligned}
\mathscr{H}(T x, T y \cap K) \leq & \varphi(d(x, y)) d(x, y) \\
& +h(y) d(g y, T x \cap K) \quad \forall x, y \in K,
\end{aligned}
$$

then $\mathscr{C} \mathscr{O} \mathscr{P}_{K}(g, T) \cap \mathscr{F}_{K}(T) \neq \emptyset$.

Proof. Since $K$ is a nonempty closed subset of $X$ and $X$ is complete, $(K, d)$ is also a complete metric space. Note first that for each $x \in K$, by (D2), we have $d(g y, T x \cap K)=0$ for all $y \in T x \cap K$. So, for each $x \in K$, by (61), we obtain

$$
d(y, T y \cap K) \leq \varphi(d(x, y)) d(x, y) \quad \forall y \in T x \cap K .
$$

Define $\kappa:[0, \infty) \rightarrow[0,1)$ by $\kappa(t)=(1+\varphi(t)) / 2$. Then, by Lemma $18, \kappa$ is also an $\mathscr{M T}$-function. Let $x \in K$ be given. Take $x_{1}=x$. Since $T x_{1} \cap K \neq \emptyset$ from (D1), we can choose $x_{2} \in T x_{1} \cap K$. If $x_{2}=x_{1}$, then $x_{1} \in \mathscr{F}_{K}(T)$ and hence $g x_{1} \in T x_{1}$ from (D2). Thus, $x_{1} \in \mathscr{C O O \mathscr { P } _ { K }}(g, T) \cap \mathscr{F}_{K}(T)$ and hence we achieved the result. Now, suppose that $x_{2} \neq x_{1}$; that is, $d\left(x_{1}, x_{2}\right)>0$. By (62), we have

$$
\begin{aligned}
d\left(x_{2}, T x_{2} \cap K\right) & \leq \varphi\left(d\left(x_{1}, x_{2}\right)\right) d\left(x_{1}, x_{2}\right) \\
& <\kappa\left(d\left(x_{1}, x_{2}\right)\right) d\left(x_{1}, x_{2}\right),
\end{aligned}
$$

which implies that there exists $x_{3} \in T x_{2} \cap K$ such that

$$
d\left(x_{2}, x_{3}\right)<\kappa\left(d\left(x_{1}, x_{2}\right)\right) d\left(x_{1}, x_{2}\right) .
$$

Next, by (62) again, there exists $x_{4} \in T x_{3} \cap K$ such that

$$
d\left(x_{3}, x_{4}\right)<\kappa\left(d\left(x_{2}, x_{3}\right)\right) d\left(x_{2}, x_{3}\right) .
$$

Iteratively, we can obtain a sequences $\left\{x_{n}\right\}$ in $K$ satisfying

$$
\begin{gathered}
x_{n+1} \in T x_{n} \cap K \\
d\left(x_{n+1}, x_{n+2}\right)<\kappa\left(d\left(x_{n}, x_{n+1}\right)\right) d\left(x_{n}, x_{n+1}\right)
\end{gathered}
$$

for each $n \in \mathbb{N}$. Since $\kappa(t)<1$ for all $t \in[0, \infty)$, by (ii), we know that $\left\{d\left(x_{n}, x_{n+1}\right)\right\}$ is strictly decreasing in $[0, \infty)$. Since $\kappa$ is an $\mathscr{M} \mathscr{T}$-function, by (g) of Theorem 7 , we obtain

$$
0<d\left(x_{1}, x_{2}\right) \leq \sup _{n \in \mathbb{N}} \kappa\left(d\left(x_{n}, x_{n+1}\right)\right)<1
$$

Let $\gamma:=\sup _{n \in \mathbb{N}} \kappa\left(d\left(x_{n}, x_{n+1}\right)\right)$. So $\gamma \in(0,1)$. By $(67)$, we have

$$
\begin{aligned}
d\left(x_{n+1}, x_{n+2}\right) & <\kappa\left(d\left(x_{n}, x_{n+1}\right)\right) d\left(x_{n}, x_{n+1}\right) \\
& \leq \gamma d\left(x_{n}, x_{n+1}\right) \\
& <\gamma^{2} d\left(x_{n-1}, x_{n}\right) \\
& <\cdots \\
& <\gamma^{n} d\left(x_{1}, x_{2}\right), \quad \text { for } n \in \mathbb{N} .
\end{aligned}
$$

Let $\alpha_{n}=\left(\gamma^{n-1} /(1-\gamma)\right) d\left(x_{1}, x_{2}\right), n \in \mathbb{N}$. For $m, n \in \mathbb{N}$ with $m>n$, we have

$$
d\left(x_{n}, x_{m}\right) \leq \sum_{j=n}^{m-1} d\left(x_{j}, x_{j+1}\right)<\alpha_{n} .
$$

Since $0<\gamma<1, \lim _{n \rightarrow \infty} \alpha_{n}=0$ and hence $\lim _{n \rightarrow \infty} \sup \left\{d\left(x_{n}, x_{m}\right): m>n\right\}=0$. This proves that $\left\{x_{n}\right\}$ is a Cauchy sequence in $K$. By the completeness of $K$, there exists $v \in K$ such that $x_{n} \rightarrow \quad v$ as $n \rightarrow \infty$. Thanks to (66) and (D2), we have

$$
g x_{n+1} \in T x_{n} \cap K \text { for each } n \in \mathbb{N} .
$$

Since $g$ is continuous and $\lim _{n \rightarrow \infty} x_{n}=v$, we have

$$
\lim _{n \rightarrow \infty} g x_{n}=g v \text {. }
$$


Since the function $x \mapsto d(x, T v)$ is continuous, by (61), (66), and (72), we get

$$
\begin{aligned}
d( & (v, T \sim K) \\
& =\lim _{n \rightarrow \infty} d\left(x_{n+1}, T v \cap K\right) \\
& \leq \lim _{n \rightarrow \infty} \mathscr{H}\left(T x_{n}, T v \cap K\right) \\
& \leq \lim _{n \rightarrow \infty}\left\{\varphi\left(d\left(x_{n}, v\right)\right) d\left(x_{n}, v\right)+h(v) d\left(g v, g x_{n+1}\right)\right\}=0,
\end{aligned}
$$

which implies $d(v, T v \cap K)=0$. By the closedness of $T v$, we have $v \in T v \cap K$. By (D2), gv $\in T v \cap K \subseteq T v$ and hence $v \in \mathscr{C} \mathscr{O} \mathscr{P}_{K}(g, T) \cap \mathscr{F}_{K}(T)$. The proof is complete.

Theorem 20. In Theorem 19, if inequality (61) is replaced with the following inequality:

$$
\begin{array}{r}
\mathscr{H}(T x, T y \cap K) \\
\leq \varphi(d(x, y)) d(x, y)+h(y) d(g y, T x) \\
\forall x, y \in K .
\end{array}
$$

Then $\mathscr{C} \mathscr{O} \mathscr{P}_{K}(g, T) \cap \mathscr{F}_{K}(T) \neq \emptyset$.

Corollary 21. Let $(X, d)$ be a complete convex metric space, $K$ a nonempty closed subset of $X, T: K \rightarrow \mathscr{B} \mathscr{C}(X)$ a multivalued map, and $g: K \rightarrow X$ a continuous self-map. Suppose that

(i) $T x \cap K \neq \emptyset$ for all $x \in K$,

(ii) $T x \cap K$ is g-invariant (i.e., $g(T x \cap K) \subseteq T x \cap K)$ for each $x \in K$,

(iii) there exist an $\mathscr{M} \mathscr{T}$-function $\varphi:[0, \infty) \rightarrow[0,1)$ and $L \geq 0$ such that

$$
\begin{aligned}
& \mathscr{H}(T x, T y \cap K) \\
& \quad \leq \varphi(d(x, y)) d(x, y)+L d(g y, T x \cap K) \quad \forall x, y \in K .
\end{aligned}
$$

Then $\mathscr{C} \mathscr{O} \mathscr{P}_{K}(g, T) \cap \mathscr{F}_{K}(T) \neq \emptyset$.

Corollary 22. Let $(X, d)$ be a complete convex metric space, $K$ a nonempty closed subset of $X, T: K \rightarrow \mathscr{B} \mathscr{C}(X)$ a multivalued map, and $g: K \rightarrow X$ a continuous self-map. Suppose that

(i) $T x \cap K \neq \emptyset$ for all $x \in K$,

(ii) $T x \cap K$ is g-invariant (i.e. $g(T x \cap K) \subseteq T x \cap K)$ for each $x \in K$,

(ii) there exist an $\mathscr{M} \mathscr{T}$-function $\varphi:[0, \infty) \rightarrow[0,1)$ and $L \geq 0$ such that

$$
\begin{aligned}
& \mathscr{H}(T x, T y \cap K) \\
& \quad \leq \varphi(d(x, y)) d(x, y)+L d(g y, T x) \quad \forall x, y \in K .
\end{aligned}
$$

Then $\mathscr{C} \mathscr{O} \mathscr{P}_{K}(g, T) \cap \mathscr{F}_{K}(T) \neq \emptyset$.

As a direct consequence of Theorems 19 and 20, we obtain the following fixed point result for multivalued non-selfmaps of Du type in complete metric spaces.
Theorem 23. Let $(X, d)$ be a complete convex metric space, $K$ a nonempty closed subset of $X$, and $T: K \rightarrow \mathscr{B} \mathscr{C}(X)$ a multivalued map. Suppose that $T x \cap K \neq \emptyset$ for all $x \in K$, and one of the following conditions holds:

(W1) there exist an $\mathscr{M} \mathscr{T}$-function $\varphi:[0, \infty) \rightarrow[0,1)$ and a function $h: K \rightarrow[0, \infty)$ such that

$$
\begin{aligned}
& \mathscr{H}(T x, T y \cap K) \\
& \quad \leq \varphi(d(x, y)) d(x, y)+h(y) d(y, T x \cap K) \quad \forall x, y \in K,
\end{aligned}
$$

(W2) there exist an $\mathscr{M} \mathscr{T}$-function $\varphi:[0, \infty) \rightarrow[0,1)$ and a function $h: K \rightarrow[0, \infty)$ such that

$$
\begin{aligned}
& \mathscr{H}(T x, T y \cap K) \\
& \quad \leq \varphi(d(x, y)) d(x, y)+h(y) d(y, T x) \quad \forall x, y \in K .
\end{aligned}
$$

Then $\mathscr{F}_{K}(T) \neq \emptyset$.

Proof. Let $g=i d$ be the identity map. It is easy to verify that all the conditions of Theorem 19 (or Theorem 20) are satisfied. Hence the conclusion follows from Theorem 19 (or Theorem 20).

The following fixed point theorems for multivalued nonself-maps of generalized Berinde-Berinde type and generalized Mizoguchi-Takahashi type are established immediately from Theorem 23.

Corollary 24. Let $(X, d)$ be a complete convex metric space, $K$ a nonempty closed subset of $X$, and $T: K \rightarrow \mathscr{B} \mathscr{C}(X) a$ multivalued map. Suppose that

(i) $T x \cap K \neq \emptyset$ for all $x \in K$,

(ii) there exist an $\mathscr{M} \mathscr{T}$-function $\varphi:[0, \infty) \rightarrow[0,1)$ and $L \geq 0$ such that

$$
\begin{aligned}
\mathscr{H} & (T x, T y \cap K) \\
& \leq \varphi(d(x, y)) d(x, y)+L d(y, T x \cap K) \quad \forall x, y \in K .
\end{aligned}
$$

Then $\mathscr{F}_{K}(T) \neq \emptyset$.

Corollary 25. Let $(X, d)$ be a complete convex metric space, $K$ a nonempty closed subset of $X$, and $T: K \rightarrow \mathscr{B} \mathscr{C}(X) a$ multivalued map. Suppose that

(i) $T x \cap K \neq \emptyset$ for all $x \in K$,

(ii) there exist an $\mathscr{M} \mathscr{T}$-function $\varphi:[0, \infty) \rightarrow[0,1)$ and $L \geq 0$ such that

$$
\begin{aligned}
& \mathscr{H}(T x, T y \cap K) \\
& \quad \leq \varphi(d(x, y)) d(x, y)+L d(y, T x) \quad \forall x, y \in K .
\end{aligned}
$$

Then $\mathscr{F}_{K}(T) \neq \emptyset$. 
Corollary 26. Let $(X, d)$ be a complete convex metric space, $K$ a nonempty closed subset of $X$, and $T: K \rightarrow \mathscr{B} \mathscr{C}(X) a$ multivalued map. Suppose that

(i) $T x \cap K \neq \emptyset$ for all $x \in K$,

(ii) there exists an $\mathscr{M} \mathscr{T}$-function $\varphi:[0, \infty) \rightarrow[0,1)$ such that

$$
\begin{aligned}
& \mathscr{H}(T x, T y \cap K) \\
& \quad \leq \varphi(d(x, y)) d(x, y) \quad \forall x, y \in K .
\end{aligned}
$$

Then $\mathscr{F}_{K}(T) \neq \emptyset$.

Remark 27. (a) If $K=X$ in Theorem 23, then we can obtain Du's fixed point theorem [12, Theorem 2.6].

(b) Theorems 19, 20 and 23, and Corollaries 21-26 all generalize and improve Du's fixed point theorem, BerindeBerinde's fixed point theorem, Mizoguchi-Takahashi's fixed point theorem, Nadler's fixed point theorem, and Banach's contraction principle.

\section{Acknowledgment}

The first author was supported partially by grant no. NSC 101-2115-M-017-001 of the National Science Council of the Republic of China.

\section{References}

[1] S. Banach, "Sur les operations dans les ensembles abstraits et leur application aux equations integerales," Fundamenta Mathematicae, vol. 3, pp. 133-181, 1922.

[2] S. B. Nadler, Jr., "Multi-valued contraction mappings," Pacific Journal of Mathematics, vol. 30, pp. 475-488, 1969.

[3] N. Mizoguchi and W. Takahashi, "Fixed point theorems for multivalued mappings on complete metric spaces," Journal of Mathematical Analysis and Applications, vol. 141, no. 1, pp. 177188, 1989.

[4] M. Berinde and V. Berinde, "On a general class of multi-valued weakly Picard mappings," Journal of Mathematical Analysis and Applications, vol. 326, no. 2, pp. 772-782, 2007.

[5] R. Kannan, "Some results on fixed points. II," The American Mathematical Monthly, vol. 76, pp. 405-408, 1969.

[6] S. K. Chatterjea, "Fixed-point theorems," Comptes Rendus de l'Académie Bulgare des Sciences, vol. 25, pp. 727-730, 1972.

[7] N. Shioji, T. Suzuki, and W. Takahashi, "Contractive mappings, Kannan mappings and metric completeness," Proceedings of the American Mathematical Society, vol. 126, no. 10, pp. 3117-3124, 1998.

[8] S. Reich, "Some problems and results in fixed point theory," in Topological Methods in Nonlinear Functional Analysis (Toronto, Ont., 1982), vol. 21 of Contemp. Math., pp. 179-187, American Mathematical Society, Providence, RI, USA, 1983.

[9] W.-S. Du, "Some new results and generalizations in metric fixed point theory," Nonlinear Analysis: Theory, Methods \& Applications, vol. 73, no. 5, pp. 1439-1446, 2010.

[10] Z. He, W.-S. Du, and I.-J. Lin, "The existence of fixed points for new nonlinear multivalued maps and their applications," Fixed Point Theory and Applications, vol. 2011, article 84, 13 pages, 2011.
[11] W.-S. Du, "On generalized weakly directional contractions and approximate fixed point property with applications," Fixed Point Theory and Applications, vol. 2012, article 6, 22 pages, 2012.

[12] W.-S. Du, "On coincidence point and fixed point theorems for nonlinear multivalued maps," Topology and Its Applications, vol. 159, no. 1, pp. 49-56, 2012.

[13] W.-S. Du, "On approximate coincidence point properties and their applications to fixed point theory," Journal of Applied Mathematics, vol. 2012, Article ID 302830, 17 pages, 2012.

[14] W.-S. Du, Z. He, and Y.-L. Chen, "New existence theorems for approximate coincidence point property and approximate fixed point property with applications to metric fixed point theory," Journal of Nonlinear and Convex Analysis, vol. 13, no. 3, pp. 459474, 2012.

[15] W. S. Du, "New cone fixed point theorems for nonlinear multivalued maps with their applications," Applied Mathematics Letters, vol. 24, no. 2, pp. 172-178, 2011.

[16] W.-S. Du and S.-X. Zheng, "Nonlinear conditions for coincidence point and fixed point theorems," Taiwanese Journal of Mathematics, vol. 16, no. 3, pp. 857-868, 2012.

[17] W.-S. Du and H. Lakzian, "Nonlinear conditions for the existence of best proximity points," Journal of Inequalities and Applications, vol. 2012, article 206, 7 pages, 2012.

[18] W.-S. Du, "New existence results and generalizations for coincidence points and fixed points without global completeness," Abstract and Applied Analysis, vol. 2013, Article ID 214230, 12 pages, 2013.

[19] F. E. Browder, "Nonexpansive nonlinear operators in a Banach space," Proceedings of the National Academy of Sciences of the United States of America, vol. 54, pp. 1041-1044, 1965.

[20] F. E. Browder and W. V. Petryshyn, "Construction of fixed points of nonlinear mappings in Hilbert space," Journal of Mathematical Analysis and Applications, vol. 20, pp. 197-228, 1967.

[21] W. A. Kirk, "Remarks on pseudo-contractive mappings," Proceedings of the American Mathematical Society, vol. 25, pp. 820$823,1970$.

[22] W. A. Kirk, "Fixed point theorems for nonlinear nonexpansive and generalized contraction mappings," Pacific Journal of Mathematics, vol. 38, pp. 89-94, 1971.

[23] N. A. Assad and W. A. Kirk, "Fixed point theorems for set-valued mappings of contractive type," Pacific Journal of Mathematics, vol. 43, pp. 553-562, 1972.

[24] S. Reich, "Fixed points of condensing functions," Journal of Mathematical Analysis and Applications, vol. 41, pp. 460-467, 1973.

[25] N. A. Assad, "On some nonself nonlinear contractions," Mathematica Japonica, vol. 33, no. 1, pp. 17-26, 1988.

[26] N. A. Assad, "On some nonself mappings in Banach spaces," Mathematica Japonica, vol. 33, no. 4, pp. 501-515, 1988.

[27] N. A. Assad, "A fixed point theorem for some non-selfmappings," Tamkang Journal of Mathematics, vol. 21, no. 4, pp. 387-393, 1990.

[28] W. Sintunavarat and P. Kumam, "Weak condition for generalized multi-valued $(f, \alpha, \beta)$-weak contraction mappings," Applied Mathematics Letters, vol. 24, no. 4, pp. 460-465, 2011.

[29] W. Sintunavarat and P. Kumam, "Common fixed point theorem for hybrid generalized multi-valued contraction mappings," Applied Mathematics Letters, vol. 25, no. 1, pp. 52-57, 2012.

[30] W. Sintunavarat and P. Kumam, "Common fixed point theorem for cyclic generalized multi-valued contraction mappings," Applied Mathematics Letters, vol. 25, no. 11, pp. 1849-1855, 2012. 


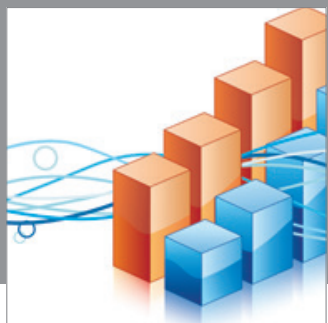

Advances in

Operations Research

mansans

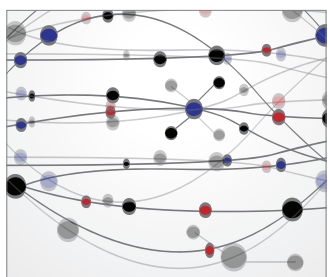

The Scientific World Journal
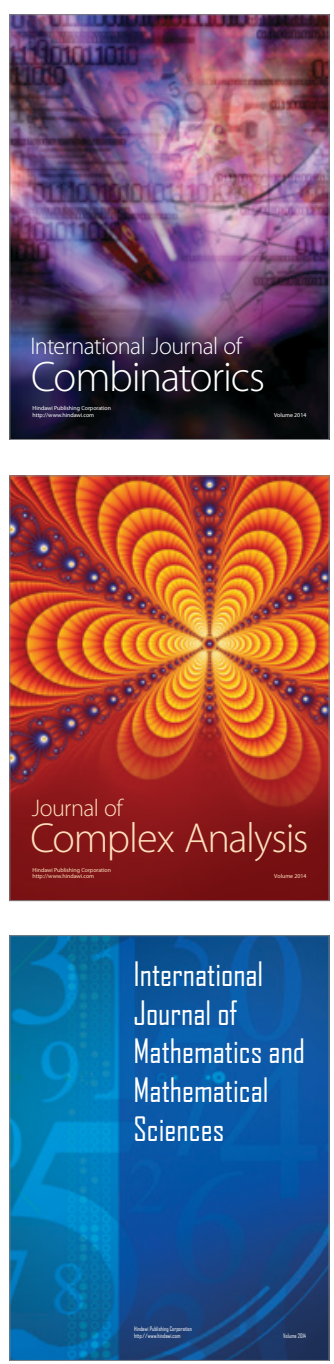
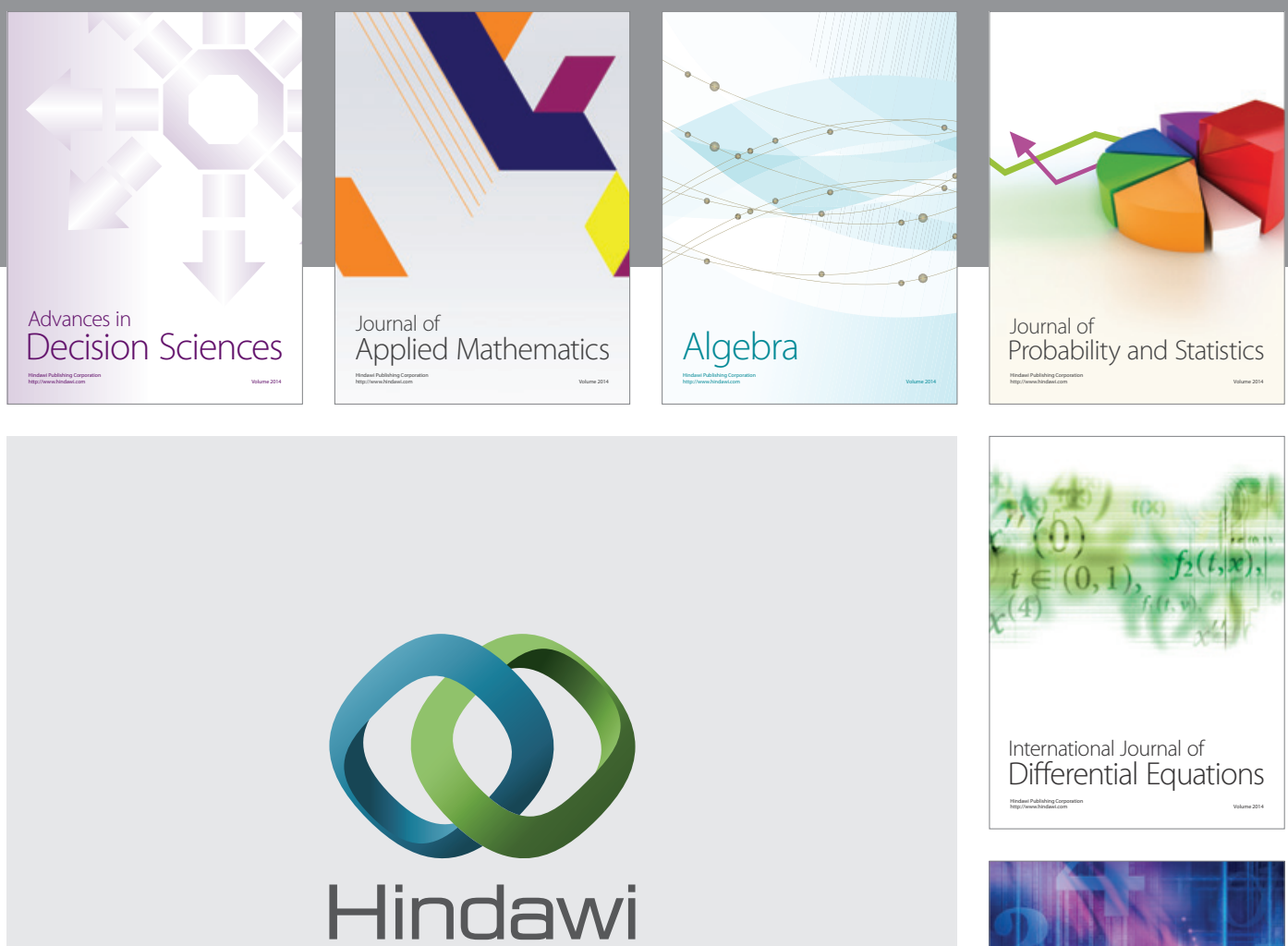

Submit your manuscripts at http://www.hindawi.com
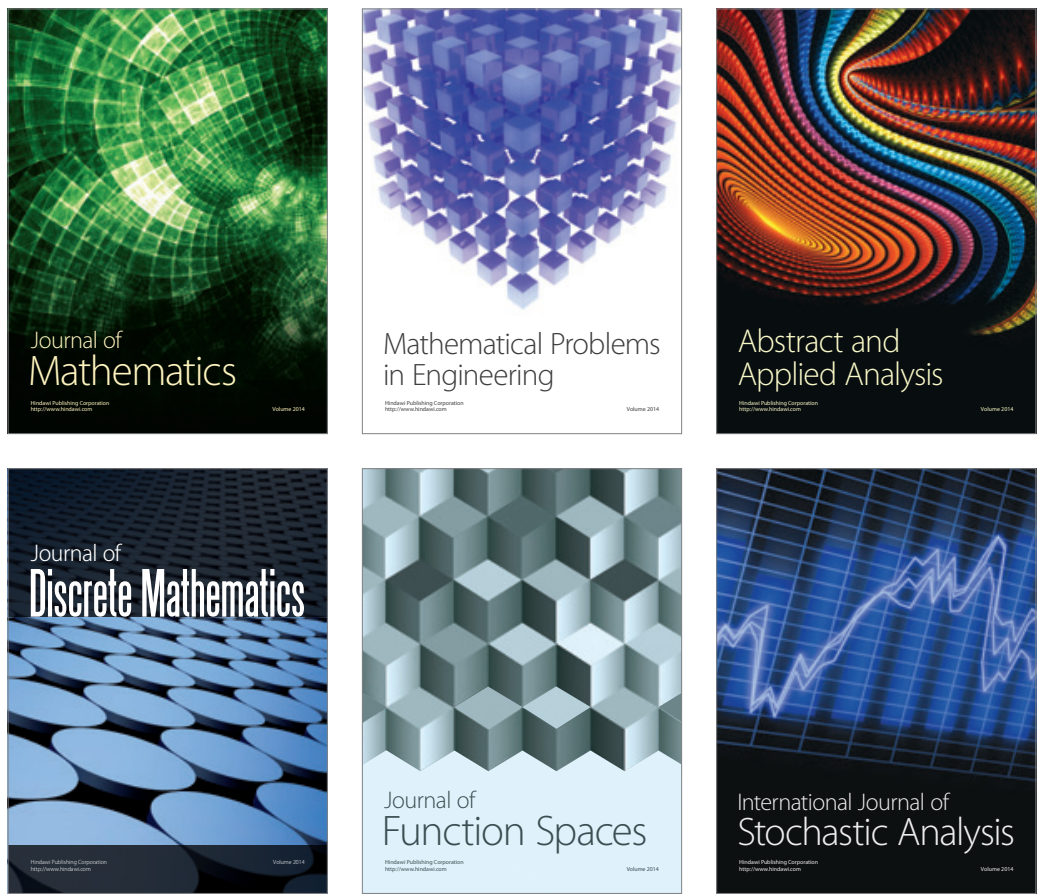

Journal of

Function Spaces

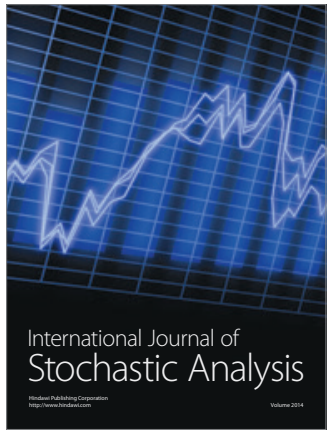

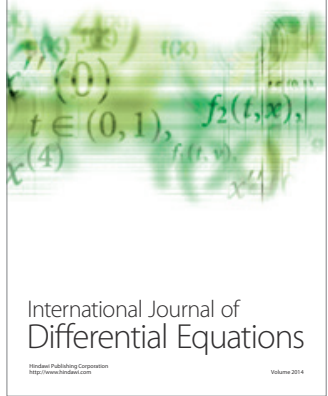
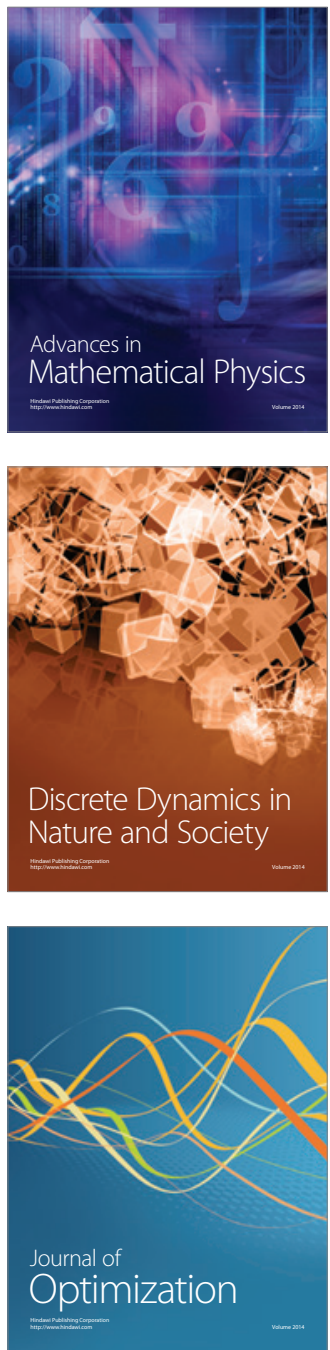